\title{
Human-Like Impedance and Minimum Effort Control for Natural and Efficient Manipulation
}

\author{
Arash Ajoudani, Marco Gabiccini, Nikos G. Tsagarakis and Antonio Bicchi
}

\begin{abstract}
Humans incorporate and switch between learnt neuromotor strategies while performing complex tasks. Towards this purpose, kinematic redundancy is exploited in order to achieve optimized performance. Inspired by the superior motor skills of humans, in this paper, we investigate a combined free motion and interaction controller in a certain class of robotic manipulation. In this bimodal controller, kinematic degrees of redundancy are adapted according to task-suitable dynamic costs. The proposed algorithm attributes high priority to minimum-effort controller while performing point to point free space movements. Once the robot comes in contact with the environment, the Tele-Impedance, common mode and configuration dependent stiffness (CMS-CDS) controller will replicate the human's estimated endpoint stiffness and measured equilibrium position profiles in the slave robotic arm, in real-time. Results of the proposed controller in contact with the environment are compared with the ones derived from Tele-Impedance implemented using torque based classical Cartesian stiffness control. The minimum-effort and interaction performance achieved highlights the possibility of adopting human-like and sophisticated strategies in humanoid robots or the ones with adequate degrees of redundancy, in order to accomplish tasks in a certain class of robotic manipulation.
\end{abstract}

\section{INTRODUCTION}

Humans incorporate and adapt various criteria while executing complex tasks. Towards this aim, the central nervous system (CNS) is deemed to prioritize among learnt optimized strategies, regarding kinematic or dynamic coordinates [1], [2]. For instance, during the reaching phase (point-topoint movements in free space with no interaction with environment), Uno et al [3] proposed a minimum joint torque change criterion and suggested that the CNS plans arm movements in dynamic coordinates rather than kinematic ones. However, suitability of such criterion will be altered by changes in task requirements (such as manipulability, interaction forces, obstacle avoidance). Consequently, neuromotor strategies will be prioritized and switched based on recent task requirements, determined by contextual information [4].

Consider a complex task in which the human arm reaching phase will be followed by an interaction with the environment. In a later phase, the desired criterion will be formed by taking into account the contact stability. Previous studies suggest that humans satisfy the requirements of such condition by means of modulation of mechanical properties

Arash Ajoudani, Marco Gabiccini and Antonio Bicchi are with Interdepartmental Research Centre "E. Piaggio", Faculty of Engineering, University of Pisa, and with the Dept. of Advanced Robotics, Istituto Italiano di Tecnologia, Via Morego 30, 16163, Genova, Italy (e-mails: arash.ajoudani@iit.it, m.gabiccini@ing.unipi.it and bicchi@centropiaggio.unipi.it).

Nikos G. Tsagarakis is with the Dept. of Advanced Robotics, Istituto Italiano di Tecnologia, Via Morego 30, 16163, Genova, Italy (e-mail: nikos.tsagarakis@iit.it). of the arm. Towards this aim, efficient restoring forces are generated in order to confront environmental displacements [5].

Static components of such mechanical property are graphically represented by stiffness ellipsoid [6]. It has been observed that the contribution of the co-activation of involved muscles in the modulation of the stiffness ellipsoid size is much more effective than adjustments in its directionality [7], [8]. Furthermore, previous studies give evidence on limited ability of humans in changing the orientation of the postural stiffness ellipsoid, even subsequent to learning stages [9]. As a result, in a fixed posture, human arm endpoint will be less stiff in certain directions than others.

In order to compensate for that, self-selected postures provide the possibility of task-oriented adjustments of the endpoint ellipsoid. Indeed, contribution of the predictive postural control to mechanical stability in humans is shown to be more effective than the role of co-contraction [7], [8]. However, postural adjustments are extremely limited by the task constraints and mainly rely on the kinematic redundancy.

Incorporation of human-like multiple-criteria strategies in the operation of the sufficiently redundant robots will result in safe and efficient robotic manipulation. For instance, by means of implementation of minimum joint torque (minimum-effort) criterion, notable amount of energy will be restored as result of predictive control of degrees of redundancy [2]. Furthermore, replication of human-like impedance regulation mechanisms in robots can permit them to safely and efficiently operate in unstructured environments under unpredicted interaction scenarios. Traditionally, the implementation of impedance regulation in robots is achieved by introduction of torque controlled robots which can regulate actively their stiffness or full impedance properties by active control techniques [10]. More recently, the development of actuation systems which inherently integrate physical principles such as variable stiffness and damping [11], [12], [13] permitted the intrinsic regulation of the robot impedance. Other studies explored the kinematic redundancy to regulate the endpoint stiffness profile. In the work of [14], based on a desired endpoint stiffness matrix, the joint stiffness levels were optimized and kept fixed. Consequently, a nullspace optimization algorithm (in a less realtime planning layer) was adopted to additionally reduce the endpoint stiffness error.

Recently, a human-like impedance controller which consists of common mode stiffness (CMS) and configuration dependent stiffness (CDS) controllers has been proposed [15]. Design of our CMS-CDS, Tele-Impedance controller is inspired by neuromotor strategies in order to account for imposed 
limitations of multi-joint impedance regulation mechanism. Towards accomplishment of the human-robot skill coordination, while being motivated by predictive switching policies of CNS [4], in this paper, we investigate the efficiency of a multiple criteria controller in robotic manipulation. The algorithm combines the human's free movement efficiency and interaction performance based on the minimization of the effect of gravity loading on the robotic joints and the error between time-varying desired stiffness matrix and the one realized at the robot endpoint, respectively. Task-oriented prioritization of the minimum-effort and CMS-CDS criteria while assuring smoothness of joint trajectories is performed by a decision rule obtained by a priority based soft switching logic. Results of the implementation of the multiple-criteria controller are experimentally evaluated in a peg in the hole task and compared to those derived from implementation based on the original Tele-Impedance control, during interaction phase. The original Tele-Impedance control [16], [17], exploited traditional Cartesian stiffness control in order to realize the desired endpoint stiffness.

The paper is structured as follows; section II briefly presents the estimation of human arm impedance in 3D space and the identification-calibration process. Section III presents the design of the minimum-effort controller, CMSCDS controller and priority based soft switching logic. The experimental setup and results of the robotic peg-inhole task by means of minimum-effort and Tele-Impedance implemented using the proposed multiple criteria controller and Cartesian impedance control are introduced in section IV and V respectively. Finally section VI addresses the conclusions.

\section{Human Impedance Modeling In 3D}

Identification of mechanical properties of the human arm is commonly performed by means of imposing known displacements to it and probing the resulting steady state force response [6], [5]. Application of such methodologies in stiffness profile estimations while executing a constrained task would be bothersome. Consequently, our previous studies were devoted to model-based human arm stiffness estimation in real-time [16], [17]. In our model, the overall mapping between muscular activities and resulting arm endpoint force $\left(T_{F}\right)$ and stiffness $\left(T_{\sigma}\right)$ in Cartesian coordinates around arm equilibrium position (close to isometric condition) is described by

$$
\left[\begin{array}{c}
F \\
\sigma
\end{array}\right]=\left[\begin{array}{c}
T_{F} \\
T_{\sigma}
\end{array}\right] P+\left[\begin{array}{c}
0 \\
\sigma_{0}
\end{array}\right],
$$

where $F$ and $\sigma$ are the endpoint force and stiffness vectors, respectively, $\sigma_{0}$ is the intrinsic stiffness in relaxed conditions, and $P \in \mathbb{R}^{n}$ is the vector of muscular activities of the $n$ considered muscles, as obtained from preprocessing EMG signals from electrodes applied on each muscle.

Now, we consider a decomposition of the vector of muscular activations $P$ in a force-generating component $P_{F}$ and a stiffness-regulating component $P_{k}$. For the first-order linearized model (1), we write

$$
P=T_{F}^{R} T_{F} P+\left(I-T_{F}^{R} T_{F}\right) P \stackrel{\text { def }}{=} P_{F}+P_{k},
$$

where $T_{F}^{R}$ denotes the generalized right-inverse of $T_{F}$, i.e. a $n \times 3$ matrix such that $T_{F} T_{F}^{R}=I$, the $3 \times 3$ identity. The stiffness-generating component $P_{k}$ is a projection of the measured EMG vector $P$ in the kernel of the force-generating map $T_{F}$. Now, let $N_{F}$ denote a basis matrix for the kernel of $T_{F}$, and let $\lambda=N_{F}^{+} P_{k}=Q P$ be the coordinates in that basis of $P_{k}$, where $Q \stackrel{\text { def }}{=} N_{F}^{+}\left(I-T_{F}^{R} T_{F}\right)$. Using this model of stiffness regulation, we write

$$
\sigma-\sigma_{0}=T_{\sigma} P=M_{\sigma} Q P
$$

where $M_{\sigma}$ denotes the mapping from the kernel of $T_{F}$ to translational stiffness profile. The map $M_{\sigma}$ needs to be identified and calibrated once, based on direct measurements of human arm end point stiffness, at different coactivation levels as described in [17]. It must be noted that the choice of a particular right-inverse does affect the coordinates of the projected vectors. However, the calibration matrix $M_{\sigma}$ is experimentally evaluated after the choice of the right inverse. Indeed, different right-inverses will lead to different $M_{\sigma}$, but eventually to the same endpoint stiffness values.

Inefficiency of the model to estimate stiffness profile in whole arm workspace (far from reference posture), forms the main drawback of the proposed method. However, since the interaction with the environment is chosen to be performed in the vicinity of the reference posture (where the model is calibrated and identified), the algorithm will provide reasonable traces of the human endpoint stiffness profile. In addition, concerning point-to-point movements, since there is no interaction with the environment, inaccuracy of the stiffness estimations will not be crucial.

Calibration-Identification of the Stiffness Model: One healthy subject (male; age 29) participated in the identificationcalibration experiments. The subject stood upright with the feet side-by-side in front of a robotic arm. The robot arm was equipped at the endpoint with a handle connected to a 6-axis force and torque (F/T) sensor (ATI Mini-45). A passive spherical joint was used to connect the center of the handle to the F/T sensor to prevent the exertion of torques by the subject (see details in [17], [16]). Displacements of the human arm at its endpoint (considered as the wrist center) were tracked by an Optitrack system (Natural Point, Inc.), with a nominal resolution of $0.02 \mathrm{~mm}$. Optical markers were also placed at the shoulder and elbow of the subject's arm. Both force and position measurements were acquired at a sampling frequency of $200 \mathrm{~Hz}$, and filtered by a low-pass Butterworth filter with cutoff frequency $15 \mathrm{~Hz}$ to eliminate noise. Eight dominant muscles acting on elbow and shoulder joints were chosen as the sources of surface electromyograms (EMGs) recordings [17]. The analogue EMG signals were measured and amplified with a Delsys-Bagnoli 16 (Delsys Inc.) apparatus. The acquired signals were band-pass filtered in the frequency range $[20,450] \mathrm{Hz}$. The resulting EMG 
signals were subsequently sampled at $2 \mathrm{kHz}$ (PCI-6220, National Instruments) and full rectified for further processing. A digital non-causal FIR linear phase low-pass filter was used for the detection of the envelope of the signal, which approximately corresponds to muscle activity.

The robotic arm used is a seven degrees-of-freedom (DoF) KUKA LWR with DLR's Fast Research (FR) Interface [18]. The data acquisition and synchronization interface between the KUKA controller, the EMG acquisition board, the Optitrack position streaming data, the six axes F/T sensor were developed in $\mathrm{C}++$.

The subject performed two identification experiments. Initially, the force-generating map $T_{F}$, was estimated and a basis of its nullspace and the projector matrix $Q$ used in (2) were computed (see [17] for details). In a second set of experiments, aimed at estimating the stiffness-regulating map $M_{\sigma}$, we applied continuous stochastic perturbations to the subject's hand through the KUKA LWR handle in $\mathrm{x}, \mathrm{y}$ and $\mathrm{z}$ directions in order to minimize voluntary stiffening behavior of the subjects arm [9].

\section{CONTROLLER DESIGN}

In this section, the bio-inspired bimodal controller, which consists of minimum-effort and CMS-CDS Tele-Impedance controllers, is described.

\section{A. Minimum-Effort Controller}

The proposed minimum effort null-space controller minimizes the effect of gravity loading on the robotic joints in reaching phase. Therefore, a cost function which incorporates joint torques as result of joint masses and the payload is defined as follows

$$
C_{g}\left(q_{e}\right)=\tau_{g}^{T} W \tau_{g},
$$

where $W, q_{e}$ and $\tau_{g}$ denote constant diagonal weighting matrix, vector of joint angles and configuration dependent gravity vector, respectively. Accordingly, concerning static conditions (relatively small endpoint velocity), the gravitational torque vector of the joint masses and the payload is obtained as follows

$$
\tau_{g}=\sum_{i=1}^{n} J_{\text {com }}{ }_{i}^{T} m_{i} g
$$

where $m, n$ and $g$ denote the joint mass (including payload), number of joints and vector of gravitational accelerations, respectively. $J_{\text {com }}$ represents the modified manipulator Jacobian and provides the relationship between joint torques and the gravitational forces, acting on the center of mass of each joint. The modified manipulator jacobian was calculated for each center of mass of the robot joints and the payload.

Optimization of above cost function (equation (3)) subject to task constraints is performed by projecting the gradient of the cost function $C_{g}$ onto the null space of the manipulator Jacobian $(J)$ [19]. Therefore we obtain

$$
\dot{q}_{e}=J^{+}\left(q_{e}\right) \dot{x}+\left[I-J^{+}\left(q_{e}\right) J\left(q_{e}\right)\right] \nabla_{q_{e}} C_{g}\left(q_{e}\right),
$$

where $J^{+}$denotes the pseudo-inverse, generally given by $J^{+}=D^{-1} J^{T}\left(J D^{-l} J^{T}\right)^{-1}$ with $D$ being a positive definite matrix, chosen to be identity here. $\nabla$ symbolizes the gradient function. By satisfying above condition in iterative way, nullspace configuration will be adapted in order to minimize the joint mass and payload gravity torque, which in the meanwhile asymptotic stability is guaranteed [19].

Similar technique can be applied for a reaching phase which will be followed by payload pickup phase, since the algorithm provides minimum-effort kinematic solution among all possible postures.

\section{B. CMS-CDS Controller}

In order to realize the human's desired endpoint stiffness profile at the slave's end effector, different strategies might be exploited. Cartesian impedance control [20] as a wellgrooved technique, establishes a mass-spring-damper relationship between the Cartesian position and force. Implementation of such control scenario requires joint torque sensing and control. Recently the development of robotic systems equipped with passive variable stiffness actuation introduced a new design paradigm towards robots with intrinsic variable joint compliance properties. In these systems, to realize the full endpoint stiffness matrix the implementation of crossjoint stiffness must be taken into account in the robot design. An alternative solution can be provided by designing a hybrid controller which exploits passive and active control [21]. In order to further investigate the problem, a human-inspired impedance controller has been proposed [15]. Design of our proposed controller is motivated by human's neuromotor strategies in order to make up for the limitations, imposed by multi-joint impedance regulation mechanism.

Previous studies, give evidence to common mode stiffness variations across the arm joints which results in severely limited ability of the humans in regulation of the directionality of the postural-stiffness ellipsoid [9], [17]. However, humans have learnt to explore and select efficient postures in order to realize the task-oriented stiffness profiles in contact with the uncertain environment [7]. Inspired by the superior interaction performance of the human arm achieved through the regulation of common mode stiffness (stiffness synergy) and self-selected posture, we suggest and design a controller which regulates the common mode stiffness across the joints in addition to a null-space controller which further minimizes the error between desired and realized endpoint stiffness.

Let $K_{c}$ be the vector of common stiffness across the joints. Inspired by the correlation of stiffness changes in human arm joint as result of co-contractions, we consider a constant scaling factor across all joints as follows

$$
K_{s}=\left[J\left(q_{c}\right)^{T} K_{h} J\left(q_{c}\right)\right]_{\text {diag }},
$$

where $q_{c}$ is the vector of joint angles and $K_{h} \in \mathbb{R}^{6 \times 6}$ is the human endpoint stiffness. The diagonal matrix $K_{s} \in$ $\mathbb{R}^{n \times n}$, with $n$ number of joints in robot, will be calculated and normalized once and used for the rest of controller design. As a result $K_{c}=k_{c m s} K_{s}$ where $k_{c m s}$ is optimized common mode stiffness in each time step. One may also 
consider optimization of constant joint scales $\left(K_{S}\right)$ based on application and task needs.

In order to realize the desired endpoint stiffness matrix $\left(K_{h}\right)$ provided by stiffness model, the following equation must be satisfied ideally

$$
K_{h} \simeq\left(J^{+}\right)^{T} K_{c} J^{+} .
$$

For the given problem we set $D=K_{c}$ as a metric tensor in the pseudo-inverse definition in section III-A. Wrong choices of the positive definite matrix $D$ in the definition of the pseudoinverse will lead in wrong estimates of the endpoint stiffness values [14]. In this case if we take the time derivative of the equation (7), we will get

$$
\begin{aligned}
\dot{K}_{h} \simeq & {\left[\left(J^{+}\right)^{T} K_{s} \dot{J}^{+}+\left(\dot{J}^{+}\right)^{T} K_{s} J^{+}\right] k_{c m s} } \\
& +\left[\left(J^{+}\right)^{T} K_{s} J^{+}\right] \dot{k}_{c m s},
\end{aligned}
$$

where two terms in first bracket of equation (8) being each others transpose. Definition of derivative of the pseudoinverse of jacobian in symbolic equation is problematic due to its complexity. However, based on a proven theorem [22], we can establish the derivative of pseudo-inverse of Jacobian matrix as follows

$$
\begin{gathered}
\frac{d J^{+}}{d t}=-J^{+} \frac{d J}{d t} J^{+}+J^{+}\left(J^{+}\right)^{T} \frac{d J^{T}}{d t} N_{J}{ }^{\perp}+ \\
{ }_{J} N^{\perp} \frac{d J^{T}}{d t}\left(J^{+}\right)^{T} J^{+},
\end{gathered}
$$

where $N_{J} \perp$ and ${ }_{J} N^{\perp}$ are the projectors on the orthogonal complement of the column and row space of $J$, respectively.

Equation (8) can be factorized with respect to $\dot{q}_{c}$. Therefore, by reshaping the equation in vector form through the operator vect( $\cdot$ ) (the operator extracts the 21 independent elements of $6 \times 6$ symmetric matrix), we get

$$
\begin{aligned}
\operatorname{vect}\left\{\dot{K}_{h}\right\} \simeq \operatorname{vect}\left\{k_{c m s}\left[\left(J^{+}\right)^{T} K_{s} A_{q_{c}}+A_{q_{c}}^{T} K_{s} J^{+}\right]\right\} \dot{q}_{c} \\
+\operatorname{vect}\left\{\left[\left(J^{+}\right)^{T} K_{s} J^{+}\right]\right\} \dot{k}_{c m s},
\end{aligned}
$$

where $A_{q_{c}}=\frac{d J^{+}}{d q_{c}}$. To simplify the expressions we can introduce $J_{q_{c}} \stackrel{\text { def }}{=} \operatorname{vect}\left\{k_{c m s}\left[\left(J^{+}\right)^{T} K_{s} A_{q_{c}}+A_{q_{c}}^{T} K_{s} J^{+}\right]\right\}$and $J_{k} \stackrel{\text { def }}{=}$ $\operatorname{vect}\left\{\left[\left(J^{+}\right)^{T} K_{s} J^{+}\right]\right\}$. Therefore we can write

$$
\dot{K}_{h}=J_{q_{c}} \dot{q}_{c}+J_{k} \dot{k}_{c m s}
$$

Now, if we take into account that $\dot{q}_{c}$ is allowed to vary in the null-space of $J$, while complying with the prescribed motion of the end-effector, we can then write

$$
\dot{q}_{c}=J^{+} K_{p} e_{p}+N_{J}^{\perp} \dot{\lambda},
$$

where $K_{p}$ is the gain matrix for Cartesian position error $e_{p}$ and $\dot{\lambda}$ a free parameter controlling the null-space velocity component. The above expression is a classical inverse kinematics problem, based on the pseudo-inverse of the Jacobian. Now, combining equation (12) and (11) we get

$$
\dot{K}_{h}=J_{q_{c}}\left(J^{+} K_{p} e_{p}+N_{J}^{\perp} \dot{\lambda}\right)+J_{k} \dot{k}_{c m s} .
$$

Defining $\dot{\tilde{K}}_{h} \stackrel{\text { def }}{=} \dot{K}_{h}-J_{q_{c}} J^{+}\left[K_{p} e_{p}\right]$ and $J_{\lambda} \stackrel{\text { def }}{=} J_{q_{c}} N_{J}{ }^{\perp}$, we can write

$$
\dot{\tilde{K}}_{h}=\left[\begin{array}{ll}
J_{\lambda} & J_{k}
\end{array}\right]\left[\begin{array}{c}
\dot{\lambda} \\
\dot{k}_{c m s}
\end{array}\right]=: J_{y} \dot{y} .
$$

Above equation resembles the structure of inverse kinematics problem of defective robots, since we are dealing with the tracking of $\dot{\tilde{K}}_{h} \in \mathbb{R}^{21}$ by using $k_{c m s}$ and degrees of kinematic redundancy. Now, by defining the vectorial stiffness error $e_{s}=\operatorname{vect}\left\{K_{h}-k_{c m s}\left[\left(J^{+}\right)^{T} K_{s} J^{+}\right]\right\}$, we can set up classical update laws. Consequently, by exploiting the update law based on the pseudo-inverse of $J_{y}$ we get

$$
\dot{y}=J_{y}{ }^{+}\left[\tilde{K}_{h}+K_{p s} e_{s}\right],
$$

where $K_{p s}$ is the gain associated to the stiffness error $e_{s}$. By implementing above update law, local asymptotic tracking will be guaranteed (see details in [15]).

Concerning design and control of soft robots, such humanlike impedance control technique will result in profitable solution since it only adopts single stiffness synergy across the arm joints. Obviously, efficiency of proposed algorithm relies on the number of degrees of kinematic redundancy. Such requirement will be satisfied by design of adequately redundant robots or by taking into account the whole body degrees of kinematic redundancy (e.g. by utilizing humanoid robots). Furthermore, once the mobility is being merged with kinematic redundancy, the efficiency of the algorithm will notably increase since extensive range of the arm postures will be accessible.

\section{Priority Based Soft Switching Logic}

In order to choose the task-related policy among minimum-effort and CMS-CDS controllers, a priority based decision rule is implemented. In the first phase of the proposed Tele-Impedance control, in which the human guides the robot's end-effector in free space, the minimum-effort null-space controller will be held responsible for control of degrees of kinematic redundancy, subject to dictated task constraints. Once the robot comes in a contact with the environment, if the interaction forces raise above a predefined value, the CMS-CDS controller takes role and $q_{c}$ will be applied ( $q_{e}$ and $q_{c}$ are estimated joint angles corresponding to the minimum-effort and CDS controllers which are calculated by equation (5) and (12), respectively). In order to produce smooth angular trajectories, a priority based soft-switching technique is defined as follows

$$
q= \begin{cases}q_{e}, & \left|F_{I}\right| \leq F_{0} \\ \xi q_{c}+(1-\xi) q_{e}, & F_{0}<\left|F_{I}\right| \leq F_{c}+F_{0} \\ q_{c}, & \left|F_{I}\right|>F_{c}+F_{0}\end{cases}
$$

where $F_{0}$ and $F_{c}$ represent the predefined forces due to noise/acceleration and minimum contact force, respectively. $F_{I}$ is formed by integration of the forces over all directions and $\xi$ is a function of force defined by

$$
\xi=\frac{F_{I}-F_{0}}{F_{c}} .
$$




\section{EXPERIMENTS}

In this section, we evaluate the effectiveness of the bimodal, bio inspired controller in a peg-in-Hole task, as a classical benchmark for spatial planning with uncertainties. As mentioned previously, the performance of the proposed methodology relies on the number of degrees of kinematic redundancy. In our experimental setup, a KUKA robotic arm with 7 joints is employed. Therefore, by taking into account a fully constrained task, only 1 degree of kinematic redundancy will be utilized for the analysis. A payload (due to the peg, FT sensor and the flange masses) was considered at the end-effector of the robot.

In our first experimental setup ${ }^{1}$, KUKA robot joint angles were commanded to an initial configuration (posture A in figure 1). Following that, the minimum-effort controller drives the robot's null-space configuration towards configuration B in which the effect of gravitational torque in robot joints is minimized. Then, the operator inserts the peg into the hole by pressing the hole along the direction of the peg. In this phase, the switching logic attributes the high priority to the CMS-CDS controller, as a response to the raise of the interaction forces. Consequently, the CMS-CDS controller drives the robot in configuration $\mathrm{C}$ in which the error between the desired full stiffness matrix and the one realized at the KUKA's end effector in minimum. In this particular experiment, a fixed, symmetric and positive definite full stiffness matrix was chosen as desired one for the CMSCDS controller. The stiffness matrix was chosen in such a way that the realized stiffness at the end effector of the robot, was high enough to overcome frictional forces between the peg and the hole.

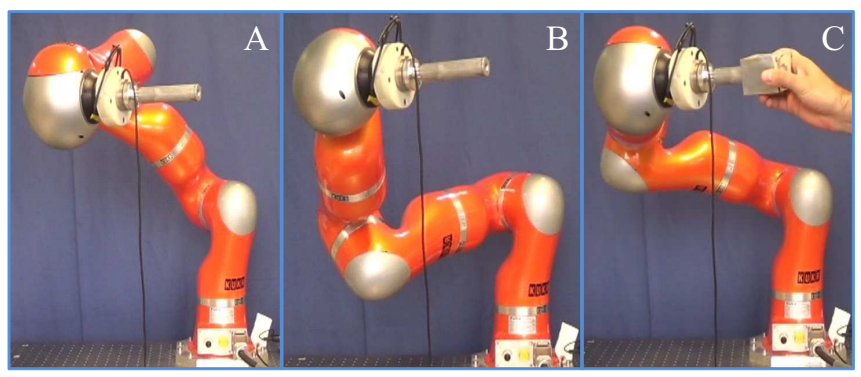

Fig. 1: KUKA robot in initial joint configuration (A), under minimum-effort (B) and CMS-CDS (C) null-space controllers.

Normalized min-effort and CMS-CDS cost functions (upper plot), interaction forces (mid plot) and joint angles (lower plot) from the experiment are depicted in Fig. 2. As it can be seen in the upper plots, once the priority based soft switching logic is turned on $(t \simeq 1 \mathrm{sec})$, the minimum-effort null-space controller takes over and reduces $C_{g}$ by approximately $\% 22$

\footnotetext{
${ }^{1}$ Comparative analysis of the stiffness matrix is generally performed by stiffness ellipsoids. However, such graphical presentation is troublesome in time-varying case. For this reason, in this work, experiments are divided in two parts with constant and time-varying desired stiffness profiles. In later case, the analysis is performed by observing the interaction forces and defining related indexes. A video of the experiment is available at [23]. Original Tele-Impedance video links are also provided [16], [17].
}

(see phase B). Such behaviour is being altered by the raise of the interaction forces $(t \simeq 7.7 \mathrm{sec}$ ). In this phase (see phase $\mathrm{C}$ ), the realized stiffness ellipsoids (translational part), by means of different stiffness control strategies (Cartesian (which also denotes desired stiffness profile), CMS-CDS and only CMS controllers) are demonstrated in Fig. (3). The 3D stiffness ellipsoids are projected in three planes. Effective changes in the orientation of the realized stiffness ellipsoid are seen once the CDS is combined with CMS (even by holding only 1 degree of kinematic redundancy in fully constrained task) which lead to $11.2 \%$ reduction in the norm of the stiffness error. Obviously, by adopting adequate degrees of redundancy (e.g. reducing the position/orient constraints or exploiting sufficiently redundant robots), the error will decrease significantly. In addition, as shown in the lower plots of Fig. 2, the priority based soft-switching technique generates smooth angular changes in the transient phase. A video of the experiment is available at [23].

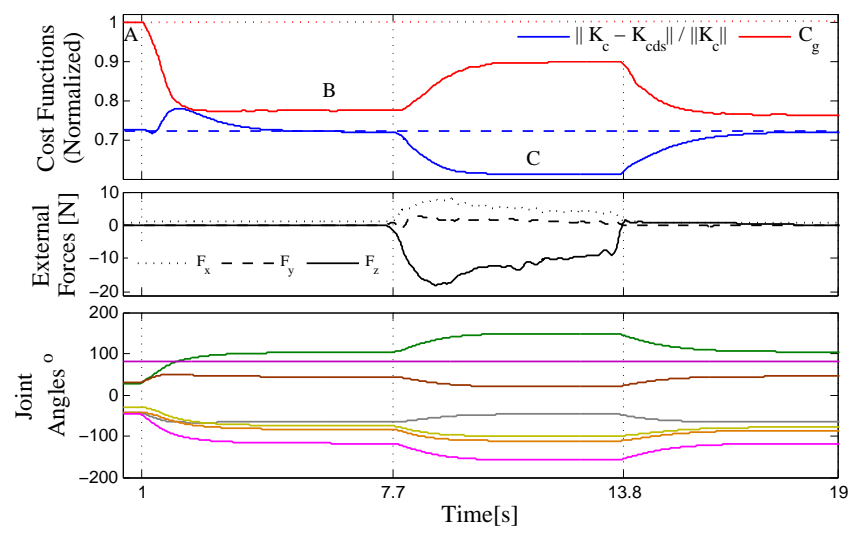

Fig. 2: Normalized minimum-effort and CMS-CDS cost functions (upper plot), controlled by priority based soft switching logic. Interaction forces and joint angles are shown in middle and lower plot, respectively. $\mathrm{A}, \mathrm{B}$ and $\mathrm{C}$ phases correspond to initial, minimumeffort and CMS-CDS joint configurations in figure 1. Dotted (red) and dashed (blue) horizontal lines in upper plot represent the mineffort and CMS-CDS cost functions without proposed control of kinematic redundancy.

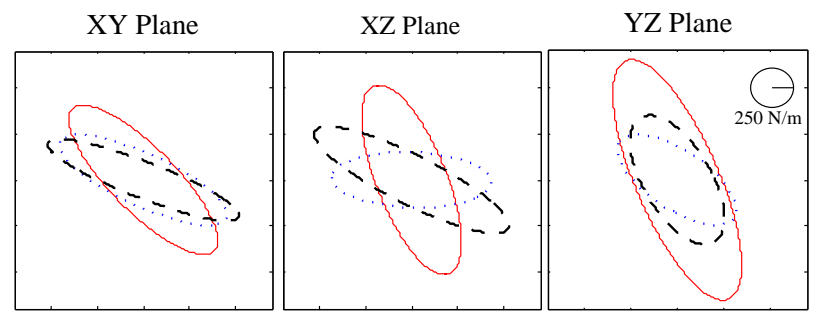

Fig. 3: Robot endpoint stiffness ellipsoids (translational) replicated by Tele-Impedance under Cartesian stiffness (solid line), CMS-CDS (broken line) and only CMS (dotted line) controllers in interaction phase. Cartesian stiffness ellipsoids coincide with the desired ones.

Second experimental setup concerns with the peg-in-hole task in a teleoperation scenario. The operator was asked to move his/her hand in the space to reach the reference 
configuration (where the stiffness model is calibrated), and finally to teleoperate the robot to insert the peg in the fixed hole. The master had visual feedback from the slave arm, including the peg and hole. No peg was hold by the master, nor did he receive any feedback except visual. Body markers were attached to the wrist in order to provide the reference trajectory for robot motion. The robot base frame was considered as the overall reference frame for other frames (Optitrack and FT sensor). The position and orientation path of the human wrist was measured, low-pass filtered (cutoff $15 \mathrm{~Hz}$ ) and used for trajectory planning. Incremental joint position references were sent to the robot, derived from the position tracking errors in six dimensions $\left(e_{p}\right)$. This approach was used to cope with drift and tracking inaccuracy due to possible delays between reference commands and generated movement in the slave end-effector. Software interfaces, sampling frequencies (processing and control), and hardware specifications are all identical to those reported in section II.

Throughout the first phase of the experiment, the master's arm endpoint stiffness profile (estimated translational and fixed rotational) along with the equilibrium trajectories were replicated in KUKA, in real-time, by means of original TeleImpedance algorithm [16], [17]. The diagonal elements of the rotational part of stiffness matrix were set to $20 \mathrm{Nm} / \mathrm{rad}$. Further development of the proposed stiffness model in section II would provide the possibility of estimation of full stiffness (rotational part) and damping matrices.

Recorded trajectories during the whole experiment were used as reference for the comparative analysis of the proposed controller. This approach was used in order to match the equilibrium path of both controllers (CMS-CDS and Cartesian) in interaction phase, while evaluating the effect of mineffort controller in reaching phase. Figure (4) demonstrates
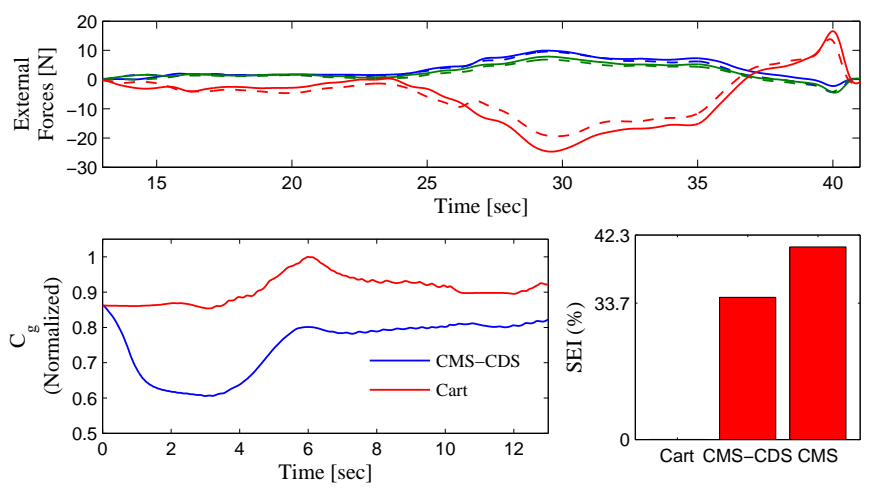

Fig. 4: Comparative analysis of Cartesian (Cart), CMS-CDS and only CMS controllers. Interaction forces along $x$ (blue), $y$ (green) and $z$ (red) for Cartesian (solid line) and CMS-CDS (dashed-line) are shown in upper plot. Lower-left plot displays the effect of mineffort controller in defined cost function $\left(C_{g}\right)$ during movement in Cartesian and CMD-CDS experiments. Lower-right plot compares the SEI index for experiments under Cartesian, CMS-CDS and only CMS controller.

the comparative analysis of described experiments. Upper graph displays the interaction forces between the peg and the hole under Cartesian and proposed CMS-CDS control.
As seen in the plots, the realized forces by two controllers are comparable. Since the equilibrium path are chosen to be the same, similarity between the interaction forces will give evidence to the approximate similarity between the realized stiffness profiles by means of both controllers. In addition, in order to numerically compare the realized stiffness error between the Cartesian and proposed controller, we define a stiffness error index as follows

$$
S E I=\frac{1}{K_{c \max }-K_{c \min }} \frac{\sum\left|K_{\text {realized }}-K_{c}\right|}{n_{p}},
$$

with $n_{p}$ being the number of data points. The above index was calculated for Cartesian, CMS-CDS and only CMS controllers for the same equilibrium path and desired stiffness profile. The average value of the calculated indexes along $x, y$ and $z$ directions are provided in Fig. (4), lower right plot. As seen in the graphs, while Cartesian controller flawlessly tracks the desired stiffness profile, application of CDS controller results in notable amount of reduction in tracking error compared to only CMS case, even by holding only 1 degree of kinematic redundancy. Results of effective reduction of gravitational torques in robot's joint due to mineffort controller in reaching phase are demonstrated in the same figure, lower left plot.

EMG variations of human arm muscles during the insertion and pull-off phase are brought in figure (5), upper plot. As noted above, during the interaction phase of the original Tele-Impedance algorithm, the stiffness profile of the human arm endpoint in reference posture is estimated and applied based on EMG signals. This time-varying stiffness profile and the equilibrium path are used as references for our proposed CMS-CDS controller. Lower plot in figure (5) demonstrates the estimated common-mode-stiffness profile in CMS-CDS experiment, with the reference stiffness profile extracted from the original Tele-Impedance experiment.

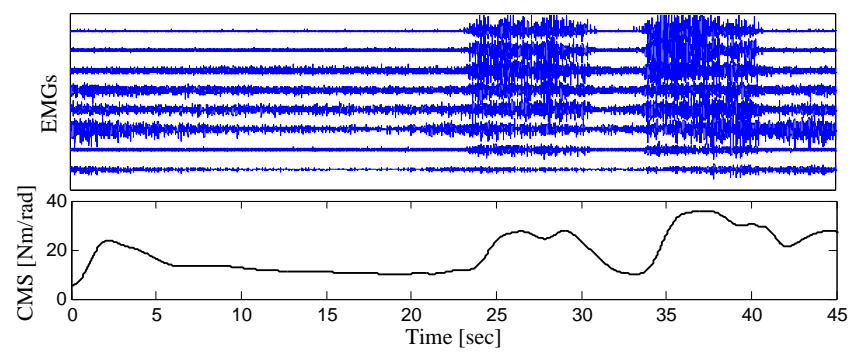

Fig. 5: Muscular activities (upper plot) and resulting commonmode-stiffness (CMS) in CMS-CDS experiment (lower plot).

It is worth noting that inspired by observations in human arm impedance regulation mechanism, the purpose of this study was to explore the single stiffness synergy across the arm joints. However, one may consider increased number of controlled joint stiffness values (CMS) to possibly improve stiffness tracking performance. Eventually, our proposed CDS controller will be adopted to further reduce the error between the desired stiffness matrix and the one realized at the robot endpoint. Such scenario will provide the possibility 
of design and control of robotic arms with $n+m$ actuators, with $n$ and $m$ being number of joints and stiffness synergies, respectively (where in this work $m=1$ ).

\section{CONCLUSIONS}

In this work, inspired by neuromotor strategies for movement and interaction efficiency, a bimodal controller, which consisted of minimum effort and CMS-CDS Tele-Impedance controllers, was developed. The initial Tele-Impedance algorithm [16], [17] provides the robot with the task-related human impedance combined with the position/orientation trajectories. This reference command is being executed under classic Cartesian impedance control. However, in the proposed multiple-criteria controller, the minimum-effort controller will minimize the effect of gravity loading on the robotic joints in reaching phase, while during the interaction with uncertain environment, the CMS and CDS are simultaneously adapting relying on the error between timevarying human's endpoint stiffness and the one, realized at robot's end-effector. The design of CMS-CDS controller was inspired by CNS policies in order to make up for imposed limitations by multi-joint impedance regulation mechanism. A priority based soft switching logic was developed to attribute the high priority to the task-suitable cost, while assuring the smoothness of the joint angles in switching phase. The energy-efficiency and interaction performance achieved highlights the possibility of adopting such sophisticated human-like strategies in humanoid robots or the ones with adequate degrees of redundancy, in order to accomplish tasks in a certain class of robotic manipulation.

\section{ACKNOWLEDGMENTS}

This work is supported by the European Community, under grants FP7 ICT-287513 "SAPHARI" and under the ERC Advanced Grant no. 291166 "SoftHands" (A Theory of Soft Synergies for a New Generation of Artificial Hands).

\section{REFERENCES}

[1] D. Wolpert, Z. Ghahramani, and M. Jordan, "Are arm trajectories planned in kinematic or dynamic coordinates? an adaptation study," Experimental brain research, vol. 103, no. 3, pp. 460-470, 1995.

[2] O. Khatib, E. Demircan, V. De Sapio, L. Sentis, T. Besier, and S. Delp, "Robotics-based synthesis of human motion," Journal of PhysiologyParis, vol. 103, no. 3, pp. 211-219, 2009.

[3] Y. Uno, M. Kawato, and R. Suzuki, "Formation and control of optimal trajectory in human multijoint arm movement," Biological cybernetics, vol. 61, no. 2, pp. 89-101, 1989.

[4] M. Kawato, "Internal models for motor control and trajectory planning," Current opinion in neurobiology, vol. 9, no. 6, pp. 718-727, 1999.

[5] E. Burdet, R. Osu, D. Franklin, T. E. Milner, and M. Kawato, "The central nervous system stabilizes unstable dynamics by learning optimal impedance," Nature, vol. 414, no. 6862, pp. 446-449, 2001.

[6] R. Osu and H. Gomi, "Multijoint muscle regulation mechanism examined by measured human arm stiffness and EMG signals," Journal of Neurophysiology, vol. 81, pp. 1458-1468, 1999.

[7] R. Trumbower, M. Krutky, B. Yang, and E. Perreault, "Use of selfselected postures to regulate multijoint stiffness during unconstrained tasks," PLoS One, vol. 4, no. 5, 2009.

[8] T. Milner, "Contribution of geometry and joint stiffness to mechanical stability of the human arm," Experimental Brain Research, vol. 143, pp. 515-519, 2002.
[9] E. Perreault, R. Kirsch, and P. Crago, "Voluntary control of static endpoint stiffness during force regulation tasks," Journal of Neurophysiology, vol. 87, pp. 2808-2816, 2002.

[10] R. Bischoff, J. Kurth, G. Schreiber, R. Koeppe, A. Albu-Schäffer, A. Beyer, O. Eiberger, S. Haddadin, A. Stemmer, G. Grunwald et al., "The kuka-dlr lightweight robot arm-a new reference platform for robotics research and manufacturing," in 41st International Symposium on on Robotics (ROBOTIK). VDE, 2010, pp. 1-8.

[11] M. Laffranchi, N. Tsagarakis, F. Cannella, and D. Caldwell, "Antagonistic and series elastic actuators: a comparative analysis on the energy consumption," in IEEE/RSJ International Conference on Intelligent Robots and Systems, 2009. IEEE, 2009, pp. 5678-5684.

[12] M. Mancini, G. Grioli, M. G. Catalano, M. Garabini, F. Bonomo, and A. Bicchi, "Passive impedance control of a qboid multi-dof vsa manipulator," in International Conference of Robotics and Automation - ICRA 2012, Saint Paul, MN, USA, May 14 - 182012.

[13] M. Grebenstein, A. Albu-Schaffer, T. Bahls, M. Chalon, O. Eiberger, W. Friedl, R. Gruber, S. Haddadin, U. Hagn, R. Haslinger et al., "The dlr hand arm system," in IEEE International Conference on Robotics and Automation (ICRA). IEEE, 2011, pp. 3175-3182.

[14] A. Albu-Schaffer, M. Fischer, G. Schreiber, F. Schoeppe, and G. Hirzinger, "Soft robotics: what cartesian stiffness can obtain with passively compliant, uncoupled joints?" in IEEE/RSJ International Conference on Intelligent Robots and Systems, 2004., vol. 4. IEEE, 2004, pp. 3295-3301.

[15] A. Ajoudani, M. Gabiccini, N. G. Tsagarakis, A. Albu-Schäffer, and A. Bicchi, "TeleImpedance: Exploring the role of common-mode and configuration-dependant stiffness," in IEEE International Conference on Humanoid Robots, 2012.

[16] A. Ajoudani, N. G. Tsagarakis, and A. Bicchi, "Tele-Impedance: Teleoperation with impedance regulation using a body-machine interface," International Journal of Robotics Research, vol. 31(13), pp. 1642-1655, 2012, http://www.youtube.com/watch?v=KPO6IO7Tr-Q.

[17] _ _ "Tele-impedance: Towards transferring human impedance regulation skills to robots," in International Conference of Robotics and Automation - ICRA 2012, Saint Paul, MN, USA, May 14 - 18 2012, http://www.youtube.com/watch?v=-Fn2dObnFpw.

[18] G. Schreiber, A. Stemmer, and R. Bischoff, "The fast research interface for the kuka lightweight robot," in IEEE Conference on Robotics and Automation (ICRA), 2010.

[19] Y. Nakamura, Advanced robotics: redundancy and optimization. Addison-Wesley Longman Publishing Co., Inc., 1990.

[20] A. Albu-Schaffer and G. Hirzinger, "Cartesian impedance control techniques for torque controlled light-weight robots," in Robotics and Automation, 2002. Proceedings. ICRA'02. IEEE International Conference on, vol. 1. IEEE, 2002, pp. 657-663.

[21] F. Petit and A. Albu-Schaffer, "Cartesian impedance control for a variable stiffness robot arm," in IEEE/RSJ International Conference on Intelligent Robots and Systems (IROS). IEEE, 2011, pp. 4180-4186.

[22] G. Golub and V. Pereyra, "The differentiation of pseudo-inverses and nonlinear least squares problems whose variables separate," SIAM Journal on numerical analysis, pp. 413-432, 1973.

[23] Video, http://www.youtube.com/watch?v=6cqu7XqIeZs. 